\title{
Impact of preoperative quality as well as quantity of skeletal muscle on survival after resection of pancreatic cancer.
}

\section{$\operatorname{AUTHOR}(S):$}

Okumura, Shinya; Kaido, Toshimi; Hamaguchi, Yuhei;

Fujimoto, Yasuhiro; Masui, Toshihiko; Mizumoto, Masaki; Hammad, Ahmed; Mori, Akira; Takaori, Kyoichi; Uemoto, Shinji

\section{CITATION:}

Okumura, Shinya ... [et al]. Impact of preoperative quality as well as quantity of skeletal muscle on survival after resection of pancreatic cancer.. Surgery 2015, 157(6): 1088-1098

\section{ISSUE DATE:}

2015-06

URL:

http://hdl.handle.net/2433/201494

\section{RIGHT:}

(c) 2015. This manuscript version is made available under the CC-BY-NC-ND 4.0 license

http://creativecommons.org/licenses/by-nc-nd/4.0/; The full-text file will be made open to the public on 30 June 2016 in accordance with publisher's 'Terms and Conditions for Self-Archiving'.; This is not the published version. Please cite only the published version.; この論文は出版社版でありません。引用の際には出版社版をご確認ご利用ください。 


\title{
Title
}

Impact of preoperative quality as well as quantity of skeletal muscle on survival after resection of pancreatic cancer.

\author{
Authors \\ Shinya Okumura, MD ${ }^{1}$ Toshimi Kaido, MD, $\mathrm{PhD},{ }^{1}$ Yuhei Hamaguchi, MD,${ }^{1}$ Yasuhiro Fujimoto, MD, \\ $\mathrm{PhD},{ }^{1}$ Toshihiko Masui, MD, PhD, ${ }^{1}$ Masaki Mizumoto, MD, PhD,${ }^{1}$ Ahmed Hammad, MD, ${ }^{1}$ Akira \\ Mori, $\mathrm{MD}, \mathrm{PhD},{ }^{1}$ Kyoichi Takaori, $\mathrm{MD}, \mathrm{PhD},{ }^{1}$ and Shinji Uemoto, $\mathrm{MD}, \mathrm{PhD} .{ }^{1}$ \\ ${ }^{1}$ Division of Hepato-Biliary-Pancreatic and Transplant Surgery, Department of Surgery, Graduate \\ School of Medicine, Kyoto University, Kyoto, Japan.
}

Correspondence to: Toshimi Kaido, $\mathrm{MD}, \mathrm{PhD}$.

Division of Hepato-Biliary-Pancreatic and Transplant Surgery, Department of Surgery, Graduate School of Medicine, Kyoto University

54 Kawahara-cho, Shogoin, Sakyo-ku, Kyoto 606-8507, Japan

Tel: +81-75-751-4323; Fax: +81-75-751-4348. E-mail: kaido@kuhp.kyoto-u.ac.jp

The category of this paper: Original communications.

Financial Support: No financial support was received from any source for this study.

Disclosure of conflicts: The authors declare that there are no conflicts of interest in relation to this study. 


\section{Abstract}

Background. Skeletal muscle depletion, referred to as sarcopenia, predicts mortality in patients undergoing digestive surgery. However, the impact of muscle quality on outcome is unclear. This retrospective study investigated the impact of preoperative skeletal muscle quantity and quality on survival in patients undergoing resection of pancreatic cancer.

Methods. We investigated 230 patients who underwent resection of pancreatic cancer between 2004 and 2013. The quantity and quality of skeletal muscle, indicated by psoas muscle mass index (PMI) and intramuscular adipose tissue content (IMAC), were measured in preoperative computed tomography images. Overall survival (OS) and recurrence-free survival (RFS) rates were compared according to PMI and IMAC, and prognostic factors after pancreatic resection were assessed.

Results. The OS and RFS rates in patients with low PMI were significantly lower than in those with normal/high PMI $(P<.001, P<.001)$, with a mean survival time (MST) of 17.7 and 33.2 months, respectively. The OS and RFS rates in patients with high IMAC were also significantly lower than in those with normal/low IMAC $(P<.001, P=.003)$ (MST $=21.5$ and 56.5 months, respectively). Low PMI (low muscle mass) and high IMAC (low muscle quality) were independent prognostic factors of poor OS (Hazard ratio $[\mathrm{HR}]=1.999, P<.001 ; \mathrm{HR}=2.527, P<.001)$ and $\mathrm{RFS}(\mathrm{HR}=1.607, P=.007$; $\mathrm{HR}=1.640, P=.004)$, respectively.

Conclusions. Preoperative sarcopenia, indicating low quality and quantity of skeletal muscle, is closely related to mortality after resection of pancreatic cancer.

(Word count of Abstract: 250 words) 


\section{Main Text}

Pancreatic cancer is common throughout the world and one of the most aggressive types of malignancy. ${ }^{1}$ Surgical resection is the only potentially curative treatment, though many patients develop recurrence after surgery. Advances in preoperative diagnosis, surgical technique, and perioperative management have improved overall and recurrence-free survival for pancreatic cancer after resection. However, even in patients who undergo curative resection, prognosis remains poor. ${ }^{2}$

In regard to prognostic factors after pancreatic resection, several reports have addressed tumorspecific factors, such as tumor size, tumor differentiation, lymph node metastasis, vascular invasion, and resection margin status. However, prognosis after surgery is multifactorial and related not only to tumorspecific factors but also individual patient characteristics. Loss of skeletal muscle mass, termed sarcopenia, has recently been identified as a poor prognostic factor in patients after digestive surgery ${ }^{3-7}$. As for those with pancreatic cancer, Peng et al. ${ }^{8}$ reported that low skeletal muscle volume was an independent risk factor for poor 3-year survival after pancreatic resection.

Sarcopenia was initially described in 1989 as an age-related decrease in muscle mass. ${ }^{9}$ The European Working Group on Sarcopenia in Older People defined the condition as a syndrome characterized by progressive and generalized loss of skeletal muscle mass and strength, with a risk of adverse outcomes such as physical disability, poor quality of life, and death, and recommended that the definition of sarcopenia should include not only low muscle mass but also low muscle function. ${ }^{10}$ However, most previous studies investigated only skeletal muscle mass to define sarcopenia, because muscle strength and function are difficult to evaluate. To the best of our knowledge, no reports regarding the relationship between quality of skeletal muscle and prognosis of pancreatic cancer patients following curative pancreatic resection have been presented.

Recently, increased intramuscular adipose tissue with aging has been identified as a potential contributor to declined muscle strength and quality. Loss of muscle strength is known to depend on both 
a decrease in muscle mass and accumulation of intramuscular adipose tissue. ${ }^{11}$ To determine the quality of skeletal muscle, Kitajima et al. evaluated skeletal muscle steatosis by measuring intramuscular adipose tissue content (IMAC) using computed tomography (CT), and found that skeletal muscle steatosis was linked to the pathogenesis and severity of non-alcoholic steatohepatitis. ${ }^{12,13}$ Thus, skeletal muscle quality has recently attracted much attention, though the impact on surgical patients remains unknown.

In the present study, we evaluated quality as well as quantity of skeletal muscle by determining IMAC and psoas muscle mass index (PMI), respectively, using preoperative plain CT imaging. We also investigated the impact of PMI and IMAC as indicators of overall quantity and quality of skeletal muscle on outcome in patients undergoing resection of pancreatic cancer.

\section{Methods}

\section{Patients and data collection}

We investigated 230 patients who underwent resection for pancreatic cancer with curative intent between January 2004 and June 2013 at Kyoto University. Clinical and pathological data were collected, including tumor characteristics and postoperative overall survival (OS) and recurrence-free survival (RFS) rates. There were 124 men (53.9\%) and 106 women (46.1\%), and their median age was 67 years (range 32-87 years). Median tumor size was $2.8 \mathrm{~cm}(0.5-7.5 \mathrm{~cm})$. Pathologocal diagnosis of all patients was pancreatic ductal adenocarcinoma. Pathological stages according to Union for International Cancer Control (UICC) staging were Ia, Ib, IIa, IIb, III, and IV in 5 (2.1\%), 8 (3.5\%), 77 (33.5\%), 129 (56.1\%), $3(1.3 \%)$ and $8(3.5 \%)$ patients, respectively. Lymph node metastasis was identified in 135 patients (58.7\%). We used 3 different types of surgical procedures; pancreaticoduodenectomy (PD) (including classic Whipple procedure, pylorus preserving pancreaticoduodenectomy, subtotal stomach preserving pancreaticoduodenectomy) $(n=155)$, distal pancreatectomy $(D P)(n=66)$, and total pancreatectomy $(n$ =7). Our current indication for resection is UICC stage $\mathrm{Ia} / \mathrm{Ib} / \mathrm{II} / \mathrm{IIb}$. Though there are some patients with 
UICC stage III/IV in this analysis, they were exceptional cases in the early period of the study, and they are not our surgical indication now. After discharge, all patients were examined for recurrence by determining tumor markers every 3 months, and by CT or magnetic resonance imaging every 6 months during the follow-up course.

\section{Image analysis}

Using preoperative plain $\mathrm{CT}$ at the umbilical level, the cross-sectional areas of the bilateral psoas muscles were measured by manual tracing, then PMI was calculated as follows.

$\mathrm{PMI}=$ cross-sectional area of bilateral psoas muscle $/$ height $^{2}\left(\mathrm{~cm}^{2} / \mathrm{m}^{2}\right)$

Low PMI was regarded as a proxy for low muscle volume (Fig 1A).

Subfascial muscular tissue in the multifidus muscle was estimated by manual tracing at the same level on preoperative plain CT images and the mean CT values [Hounsfield units $(\mathrm{HU})$ ] for these areas were determined using the Aquarius NET server (Tera Recon, Inc., San Mateo, CA, USA). We placed 4 circles on areas of subcutaneous fat away from major vessels in CT images, which were used as the region of interest (ROI) of subcutaneous fat. The mean CT values (HU) for the ROI of subcutaneous fat were also measured. IMAC was calculated by the ratio of these CT values, as previously reported by Kitajima, as follows. ${ }^{12,13}$

IMAC $=$ mean CT value of ROI of multifidus muscle $(\mathrm{HU}) /$ mean CT value of ROI of subcutaneous fat (HU).

High IMAC was regarded as a proxy for low muscle quality (Fig 1B).

\section{Cut-off values for PMI and IMAC}

Because the range of PMI in males and females is quite different, we established different cut-off lines for each using receiver operating characteristic (ROC) curves. The cut-off values were selected on the 
basis of best accuracy in relation to an outcome (death). The cut-off values for PMI in males and females were $5.896 \mathrm{~cm}^{2} / \mathrm{m}^{2}$ [area under the curve $\left.(\mathrm{AUC})=0.633\right]$ and $4.067 \mathrm{~cm}^{2} / \mathrm{m}^{2}(\mathrm{AUC}=0.567)$, respectively Based on these cut-off values, patients were assigned to 1 of 2 groups, low PMI $(n=64)$ and normal/high PMI $(n=166)$. The cut-off values for IMAC in males and females were $-0.343($ AUC $=0.626)$ and 0.256 (AUC $=0.610$ ), respectively. Based on these cut-off values, patients were assigned to 1 of 2 groups, high IMAC $(\mathrm{n}=142)$ and normal/low IMAC $(\mathrm{n}=88)$. Patient-related factors, tumor-related factors, surgical variables, postoperative complications, and rate of completion of chemotherapy were compared according to PMI and IMAC.

\section{Analyzed parameters}

The OS and RFS rates after pancreatic resection were investigated in patients classified as PMI or IMAC. Prognostic factors were examined with respect to those rates using the following variables: skeletal muscle mass (low PMI vs. normal/high PMI), skeletal muscle quality (high IMAC vs. normal/low IMAC), age ( $>70$ years vs. $\leq 70$ years), sex (male vs. female), Body mass index (BMI) $(<$ $20.0 \mathrm{~kg} / \mathrm{m}^{2}$ vs. $\left.\geq 20.0 \mathrm{~kg} / \mathrm{m}^{2}\right)$, neutrophil-lymphocyte ratio (NLR) $(>4$ vs. $\leq 4)$, platelet-lymphocyte ratio (PLR) (> 300 vs. $\leq 300)$, total lymphocyte count $($ TLC) $(<1000 / \mu \mathrm{l}$ vs. $\geq 1000 / \mu \mathrm{l})$, C-reactive protein (CRP) level ( $>1.0 \mathrm{mg} / \mathrm{dl}$ vs. $\leq 1.0 \mathrm{mg} / \mathrm{dl})$, modified Glasgow prognostic score (mGPS) (2 vs. 0/1), prognostic nutritional index (PNI) (< 45 vs. $\geq 45)$, Carbohydrate antigen 19-9 (CA19-9) level (> 200 $\mathrm{U} / \mathrm{ml} \mathrm{vs.} \leq 200 \mathrm{U} / \mathrm{ml}$ ), tumor size $(>2.0 \mathrm{~cm}$ vs. $\leq 2.0 \mathrm{~cm}$ ), tumor differentiation (poorly differentiated vs. well/moderately differentiated adenocarcinoma), lymphatic vessel invasion (LY 2/3 vs. 0/1), blood vessel invasion (V 2/3 vs. 0/1), perineural invasion (NE 2/3 vs. 0/1), nodal metastasis, curability (R1 vs. $\mathrm{R} 0$ resection), operative procedure (PD/TP vs. DP), postoperative complications (presence vs. absence), and adjuvant chemotherapy (presence vs. absence). Modified GPS was calculated as previously described. ${ }^{14}$ Briefly, patients with both elevated CRP $(>1.0 \mathrm{mg} / \mathrm{dl})$ and hypoalbuminemia $(<3.5 \mathrm{~g} / \mathrm{dl})$ 
were given a score of 2 , patients with elevated CRP and without hypoalbuminemia a score of 1 , and those with normal CRP a score of 0 . PNI was calculated as $10 \times$ albumin $(\mathrm{g} / \mathrm{dl})+0.005 \times$ total lymphocyte $\operatorname{count}(/ \mu \mathrm{l}) .{ }^{15}$ Postoperative complications were classified according to Clavien-Dindo classification, ${ }^{16}$ with complications classified as grade 3 or greater considered to indicate the presence of postoperative complications. Adjuvant chemotherapy was performed with gemcitabine, S-1, or both, with a duration over 6 months considered as the presence of adjuvant chemotherapy. Resection curability was classified as R1 (complete resection with no grossly visible tumor as defined by the surgeon, but margins microscopically positive according to pathologist) and R0 (complete resection with no microscopic residual tumor). All of these parameters could be retrospectively extracted from patient's clinical records.

\section{Statistical analysis}

Data are presented as the mean \pm standard deviation (SD) for continuous variables. Continuous variables were non-parametrically analyzed using a Mann-Whitney U test. Categorical variables were compared using a $\chi^{2}$ test or Fisher's exact test as appropriate. Any variable with $P<.10$ in univariate analysis using the above tests was considered to be a candidate for multivariate analysis using the Cox proportional hazard model. Cumulative OS and RFS rates were calculated using the Kaplan-Meier method, and differences between curves were evaluated using a log-rank test. A $P$ value $<.05$ was considered to indicate significance. All statistical data were generated using JMP 11 (SAS Institute, Cary, NC, USA) and Prism 6 (GraphPad Software, Inc., La Jolla, CA, USA).

\section{Results}

\section{Correlations of PMI and IMAC with patient age}

PMI in males $\left(7.132 \mathrm{~cm}^{2} / \mathrm{m}^{2}\right.$, range 1.717-13.943) was significantly higher than that in females (4.999 $\mathrm{cm}^{2} / \mathrm{m}^{2}, 1.906$ to 11.335$)(P<.001)($ Fig $2 \mathrm{~A})$, while IMAC in males $(-0.333,-0.777$ to +0.077$)$ was 
significantly lower than that in females $(-0.182,-0.469$ to +0.284$)(P<.001)$ (Fig 2B). PMI was significantly inversely correlated with age in males $(P=.002, \mathrm{r}=-0.280)$, while there was no correlation in females $(P=.513, \mathrm{r}=0.065)($ Fig $2 \mathrm{C})$, and IMAC was positively correlated with age in both males $(P<.001, \mathrm{r}=0.490)$ and females $(P<.001, \mathrm{r}=0.348)($ Fig $2 \mathrm{D})$. There was no significant correlation observed between PMI and IMAC (males $P=.372, \mathrm{r}=-0.081$; females $P=.277, \mathrm{r}=0.107$ ).

\section{Baseline characteristics of patients classified according to PMI}

Clinicopathological characteristics of patients with low and normal/high PMI are shown in Table I. Patients with low PMI were significantly older than those with normal/high PMI $(P=.002)$, while their body mass index $(\mathrm{BMI})$ was significantly lower $(P<.001)$. There were no significant differences between the low and normal/high PMI groups in regard to other patient-related factors, tumor-related factors, surgical variables, postoperative complications, and preoperative neo-adjuvant chemotherapy. However, the completion rate of postoperative adjuvant chemotherapy was significantly lower in patients with low PMI $(65.6 \%$ vs. $80.1 \%, P=.021)$. The reasons of the cessation of chemotherapy in patients with low PMI were patient's poor general conditions $(n=10 ; 45.5 \%)$, adverse effects of chemotherapy $(\mathrm{n}=2 ; 9.1 \%)$, and patient's advanced age $(\mathrm{n}=2 ; 9.1 \%)$.

\section{Baseline characteristics of patients classified according to IMAC}

Clinicopathological characteristics of patients with high and normal/low IMAC are shown in Table II. Patients with high IMAC were significantly older than those with normal/low IMAC $(P=.001)$, while their BMI was significantly higher $(P=.007)$. There were no significant differences between the high and normal/low IMAC groups in regard to the other factors except for tumor size. Furthermore, the completion rate of postoperative adjuvant chemotherapy was significantly lower in patients with high IMAC $(69.0 \%$ vs. $87.5 \%, P=.001)$. The reasons of the cessation of chemotherapy in patients with high 
IMAC were patient's poor general conditions $(n=26 ; 59.1 \%)$, adverse effects of chemotherapy $(n=4$; 9.1\%), and patient's advanced age $(n=4 ; 9.1 \%)$.

\section{Overall and recurrence-free survival rates after pancreatic resection}

OS and RFS curves for patients with low PMI and normal/high PMI, and with high IMAC and normal/low IMAC are shown in Fig 3. OS rates in patients with low PMI were significantly lower than in those with normal/high PMI $(P<.001)$ (Fig 3A). The mean survival time (MST) was 17.7 months and 33.2 months, respectively. OS rates in patients with high IMAC were significantly lower than in those with normal/low IMAC $(P<.001)$ (Fig 3B). MST was 21.5 months and 56.5 months, respectively. RFS rates in patients with low PMI were significantly lower than in those with normal/high PMI (P $<$.001) (Fig 3C). RFS rates in patients with high IMAC were significantly lower than in those with normal/low $\operatorname{IMAC}(P=.003)($ Fig 3D).

The patients with normal-high PMI can be divided into two groups; those with high IMAC and those with normal-low IMAC. The OS rate for patients with normal-high PMI/ high IMAC was significantly lower than that for those with normal-high PMI/ normal-low IMAC $(P<.001)$ (Fig 4A). The RFS rate for patients with normal-high PMI/ high IMAC was significantly lower than that for those with normalhigh PMI/ normal-low IMAC ( $P=.046)$ (Fig 4B). On the other hand, as for patients with low PMI, the OS and RFS rates for patients with high IMAC/ low PMI were lower than those for patients with normallow IMAC/ low PMI, while these difference were not statistically significant (OS: $P=.087$, RFS: $P$ $=.076$, respectively) .

A total of 141 patients died during the follow-up period. The cause of death in 55 patients with low PMI were as follows: primary disease $(n=50)$, sepsis $(n=2)$, cerebral infarction $(n=1)$, and others (n =2). The cause of 86 patients with normal/high PMI were as follows: primary disease $(n=81)$, myocardial infarction $(n=1)$, pneumonia $(n=1)$, other site of cancer $(n=1)$, and others $(n=2)$. There 
were no significant differences for causes of death between the 2 subgroups $(P=.448)$. Similarly, between the 2 subgroups classified according to IMAC, we found no significant differences in regard to causes of death $(P=.214)$.

\section{Risk factors for poor outcome in patients undergoing pancreatic resection}

In univariate analysis, low PMI (lower skeletal muscle mass), high IMAC (lower skeletal muscle quality), lower PNI, higher CA19-9 level, tumor size, lymphatic vessel invasion, perineural invasion, nodal metastasis, $\mathrm{R} 1$ resection, and absence of adjuvant chemotherapy were identified as significant risk factors for death after pancreatic resection for pancreatic cancer. Multivariate analysis identified low PMI, high IMAC, male gender, poorly differentiated tumor, lymphatic vessel invasion, nodal metastasis, R1 resection, and absence of adjuvant chemotherapy as 8 independent significant risk factors for death after pancreatic resection for pancreatic cancer (Table III).

Univariate analysis also revealed that low PMI, high IMAC, lower PNI, higher CA19-9 level, tumor size, lymphatic vessel invasion, perineural invasion, nodal metastasis, and R1 resection were significant risk factors for pancreatic cancer recurrence after the operation. Multivariate analysis identified low PMI, high IMAC, nodal metastasis, and R1 resection as 4 independent risk factors for pancreatic cancer recurrence after the operation (Table IV).

Thus, lower skeletal muscle mass and lower skeletal muscle quality were both identified as independent risk factors for OS and RFS.

\section{Discussion}

The results of our retrospective study revealed that preoperative PMI and IMAC are independent risk factors for mortality after curative resection of pancreatic cancer. The previous report regarding the relationship between sarcopenia and pancreatic cancer focused only on skeletal muscle mass ${ }^{8}$. To the 
best of our knowledge, this is the first study to investigate the impact of skeletal muscle quality on survival after pancreatic cancer resection.

In previous reports, only skeletal muscle mass was used to define sarcopenia, because muscle strength and function are difficult to evaluate. Indeed, it is considered that muscle mass and strength are highly correlated, and that loss of muscle strength is a direct result of lost muscle mass. However, some patients may have lower levels of muscle function and strength even though muscle mass seems to be adequate, because of reduced muscle quality. High IMAC indicates a lower level of lean muscle mass as well as higher level of intramuscular adipose tissue. Therefore, we used intramuscular adipose tissue accumulation with PMI to evaluate preoperative sarcopenia. Several studies have shown that loss of muscle tissue in sarcopenia is accompanied by infiltration with fat and connective tissue, a condition known as myosteatosis. ${ }^{17}$ Assessments of skeletal muscle quality and intramuscular fat infiltration are therefore crucial for considering sarcopenia.

In our study, though patients with normal-high PMI were considered to have normal muscle volume, patients with low muscle quality in this group had a high risk for poor survival. The OS and RFS rates for patients with normal-high PMI/ high IMAC were significantly lower than those for patients with normal-high PMI/ normal-low IMAC $(P<.001, P=.046$, respectively), which indicates that patient prognosis is highly dependent on the quality of skeletal muscle and those with low muscle quality have a poor prognosis even if muscle volume is near normal. Thus, assessment of only muscle volume is not sufficient, and evaluations of both quality and quantity of skeletal muscle are more reliable for predicting prognosis.

Following resection of pancreatic cancer, prognosis was shown to depend on several tumor-specific factors including tumor size, tumor differentiation, lymph node metastasis, and resection margin status. However, prognosis after surgery is multifactorial, and related to not only tumor-specific factors but also individual patient characteristics. Moreover, most tumor-specific factors can only be determined after 
surgery. Therefore, it is useful to reveal patient-specific potential prognostic indicators that are available before surgery. Previous studies have suggested that preoperative parameters, such as CRP, ${ }^{18}$ thrombocytosis, ${ }^{19}$ lymphocytopenia, ${ }^{20,21} \mathrm{NLR},{ }^{22,23} \mathrm{PLR}^{24}, \mathrm{mGPS}^{14}$, and $\mathrm{PNI},{ }^{15}$ represent potential prognostic markers in resected pancreatic cancer cases. In our study, tumor-specific factors including tumor differentiation, lymphatic vessel invasion, lymph node metastasis, and resection curability, as well as adjuvant chemotherapy were associated with survival. On the other hand, preoperative parameters, such as BMI, NLR, PLR, CRP, mGPS, and PNI did not remain as independent poor prognostic factors in multivariate analysis. Therefore, in terms of preoperative patient-specific factors, PMI and IMAC would be more reliable than the other preoperative markers examined in our study.

The molecular mechanism of sarcopenia remains poorly understood. Recently, skeletal muscle and adipose tissue have become to be considered as secretary organs, ${ }^{25}$ with several different cytokines and peptides (classified as myokines), such as interleukin-6 (IL-6) and insulin-like growth factor-1 (IGF-1), shown to be released from skeletal muscle, and several adipocytokines (called adipokines), such as adiponectin and leptin, released from adipose tissue. ${ }^{25}$ Myokines are considered to balance and counteract the effects of adipokines. Furthermore, the associations among immunity, inflammation, and adipocytokines have recently been emphasized as key mechanisms by which sarcopenia affects patient survival. $^{17,26}$

We are currently implementing preoperative and postoperative nutritional and exercise therapeutic protocols, especially for patients with sarcopenia or with poor nutritional status. Although the ideal period of these perioperative therapy before and after operation is not established, in our opinion, at least one month before operation and a few months after operation are required to improve sarcopenia. However, the duration of preoperative intervention should be decided incorporating the progression of pancreatic cancer during such intervention. Our group previously reported the impact of nutritional therapy on prognosis after living-donor liver transplantation, especially in patients with sarcopenia. ${ }^{7} \mathrm{We}$ 
trust that our supportive therapy will also improve the prognosis of pancreatic cancer patients, though whether nutritional and exercise therapy is truly effective must be clarified in a prospective study.

Preoperative PMI and IMAC can be very reliable parameters for prognosis after surgery. In addition, risk stratification for sarcopenia can be easily performed before surgery using these parameters. Such risk stratification and scoring would be useful for making clinical decisions, and also when considering indication of surgery for high-risk patients, though further investigations are needed. At present, we would rather make supportive interventions for high-risk patients than change our surgical indication for potentially resectable pancreatic cancer.

There are several limitations to our study. First, the correlation of IMAC and skeletal muscle strength could not be investigated because of the retrospective study design. However, recent investigations have shown that intramuscular fat accumulation contributes to declines in muscle strength and quality. ${ }^{11,27}$ Higher attenuation value, or density, of skeletal muscle shown by CT was reported to be associated with greater muscle strength independent of muscle volume. ${ }^{28}$ These findings support our notion that IMAC is a possible new parameter to assess sarcopenia instead of solely measuring muscle strength. In addition, for evaluation of sarcopenia, we are now conducting a prospective study to examine other parameters of muscle function, such as grip strength, walking speed, and levels of exhaustion, in which we hope to reveal the relationship between IMAC and muscle strength. Because of the retrospective study design, we couldn't investigate the extent of preoperative body weight loss from patient charts. We would like to prospectively investigate the relationship between preoperative body weight loss and sarcopenia markers. As for measurements using CT images, we chose to utilize the psoas muscle as a means to evaluate skeletal muscle mass and the multifidus muscle to evaluate skeletal muscle quality at the umbilical level in cross-sectional CT images, as previously reported; however, other approaches have been advocated (e.g., total skeletal muscle mass at L3 level). ${ }^{4,29}$ Another point we have to keep in mind is that the completion rate of adjuvant chemotherapy in patients with low PMI was significantly lower 
than that of those with normal/high PMI (65.6\% vs. $80.1 \%)$, and the rate in patients with high IMAC was significantly lower than that in those with normal/low IMAC (69.0\% vs. 87.5\%), which might have influenced patient survival to some extent. In patients with sarcopenia and advanced age, we are often obliged to abandon chemotherapy because of their poor general condition and severe adverse effects. However, in multivariate analysis, low PMI and high IMAC remained as independent prognostic factors for poor survival even after adjustment for the influence of chemotherapy. Finally, it is important to consider whether our cut-off values for PMI and IMAC are adequate to define sarcopenia. Several previous reports utilized separate definitions for sarcopenia, though there remains no consensus or objective criteria to define the condition. In the present study, we determined cut-off values for PMI and IMAC based on ROC curves, which is considered to be a more accurate and objective method than use of SD for setting cut-off values. However, further investigations are necessary.

In conclusion, preoperative sarcopenia, defined by both quality and quantity of skeletal muscle, was found to be closely involved with postoperative survival (OS, RFS) in patients undergoing resection of pancreatic cancer. Assessment of sarcopenia using PMI and IMAC can be easily performed. Along with postoperative tumor-specific prognostic factors, preoperative evaluation of sarcopenia, including muscle quality as well as muscle mass, may be useful for risk stratification and clinical decision-making for patients with pancreatic cancer.

\section{References}

1. Siegel R, Naishadham D, Jemal A. Cancer statistics, 2013. CA Cancer J Clin 2013;63: 11-30.

2. Conroy T, Desseigne F, Ychou M, Bouche O, Guimbaud R, Becouarn Y, et al. FOLFIRINOX versus gemcitabine for metastatic pancreatic cancer. N Engl J Med 2011;364: 1817-25.

3. Masuda T, Shirabe K, Ikegami T, Harimoto N, Yoshizumi T, Soejima Y, et al. Sarcopenia is a 
prognostic factor in living donor liver transplantation. Liver Transpl 2014;20: 401-7.

4. Harimoto N, Shirabe K, Yamashita YI, Ikegami T, Yoshizumi T, Soejima Y, et al. Sarcopenia as a predictor of prognosis in patients following hepatectomy for hepatocellular carcinoma. Br J Surg 2013;100: 1523-30.

5. van Vledder MG, Levolger S, Ayez N, Verhoef C, Tran TC, Ijzermans JN. Body composition and outcome in patients undergoing resection of colorectal liver metastases. Br J Surg 2012;99: 550-7.

6. Peng PD, van Vledder MG, Tsai S, de Jong MC, Makary M, Ng J, et al. Sarcopenia negatively impacts short-term outcomes in patients undergoing hepatic resection for colorectal liver metastasis. HPB (Oxford) 2011;13: 439-46.

7. Kaido T, Ogawa K, Fujimoto Y, Ogura Y, Hata K, Ito T, et al. Impact of sarcopenia on survival in patients undergoing living donor liver transplantation. Am J Trasnplant 2013;13: 1549-56.

8. Peng P, Hyder O, Firoozmand A, Kneuertz P, Schulick RD, Huang D, et al. Impact of sarcopenia on outcomes following resection of pancreatic adenocarcinoma. J Gastrointest Surg 2012;16: 1478-86.

9. Rosenberg IH. Sarcopenia: origins and clinical relevance. J Nutr 1997;127: 990s-1s.

10. Cruz-Jentoft AJ, Baeyens JP, Bauer JM, Boirie Y, Cederholm T, Landi F, et al. Sarcopenia: European consensus on definition and diagnosis: Report of the European Working Group on Sarcopenia in Older People. Age Ageing 2010;39: 412-23.

11. Marcus RL, Addison O, Kidde JP, Dibble LE, Lastayo PC. Skeletal muscle fat infiltration: impact of age, inactivity, and exercise. J Nutr Health Aging 2010;14: 362-6.

12. Kitajima Y, Eguchi Y, Ishibashi E, Nakashita S, Aoki S, Toda S, et al. Age-related fat deposition in multifidus muscle could be a marker for nonalcoholic fatty liver disease. J Gastroenterol 2010;45: 21824.

13. Kitajima Y, Hyogo H, Sumida Y, Eguchi Y, Ono N, Kuwashiro T, et al. Severity of non-alcoholic steatohepatitis is associated with substitution of adipose tissue in skeletal muscle. J Gastroenterol Hepatol 
2013;28: 1507-14.

14. Proctor MJ, Morrison DS, Talwar D, Balmer SM, O'Reilly DS, Foulis AK, et al. An inflammationbased prognostic score (mGPS) predicts cancer survival independent of tumour site: a Glasgow Inflammation Outcome Study. Br J Cancer 2011;104: 726-34.

15. Sun K, Chen S, Xu J, Li G, He Y. The prognostic significance of the prognostic nutritional index in cancer: a systematic review and meta-analysis. J Cancer Res Clin Oncol 2014;140: 1537-49.

16. Dindo D, Demartines N, Clavien P-A. Classification of Surgical Complications. Ann Surg 2004;240: 205-13.

17. Zoico E, Corzato F, Bambace C, Rossi AP, Micciolo R, Cinti S, et al. Myosteatosis and myofibrosis: relationship with aging, inflammation and insulin resistance. Arch Gerontol Geriatr 2013;57: 411-6.

18. Sanjay P, de Figueiredo RS, Leaver H, Ogston S, Kulli C, Polignano FM, et al. Preoperative serum C-reactive protein levels and post-operative lymph node ratio are important predictors of survival after pancreaticoduodenectomy for pancreatic ductal adenocarcinoma. JOP 2012;13: 199-204.

19. Suzuki K, Aiura K, Kitagou M, Hoshimoto S, Takahashi S, Ueda M, et al. Platelets counts closely correlate with the disease-free survival interval of pancreatic cancer patients. Hepato-gastroenterology 2004;51: 847-53.

20. Fogar P, Sperti C, Basso D, Sanzari MC, Greco E, Davoli C, et al. Decreased total lymphocyte counts in pancreatic cancer: an index of adverse outcome. Pancreas 2006;32: 22-8.

21. Yamaguchi K, Noshiro H, Shimizu S, Morisaki T, Chijiiwa K, Tanaka M. Long-term and short-term survivors after pancreatectomy for pancreatic cancer. Int Surg 2000;85: 71-6.

22. Bhatti I, Peacock O, Lloyd G, Larvin M, Hall RI. Preoperative hematologic markers as independent predictors of prognosis in resected pancreatic ductal adenocarcinoma: neutrophil-lymphocyte versus platelet-lymphocyte ratio. Am J Surg 2010;200: 197-203.

23. Wang DS, Luo HY, Qiu MZ, Wang ZQ, Zhang DS, Wang FH, et al. Comparison of the prognostic 
values of various inflammation based factors in patients with pancreatic cancer. Med Oncol 2012;29: 3092-100.

24. Smith RA, Bosonnet L, Raraty M, Sutton R, Neoptolemos JP, Campbell F, et al. Preoperative platelet-lymphocyte ratio is an independent significant prognostic marker in resected pancreatic ductal adenocarcinoma. Am J Surg 2009;197: 466-72.

25. Pedersen BK, Febbraio MA. Muscles, exercise and obesity: skeletal muscle as a secretory organ. Nat Rev Endocrinol 2012;8: 457-65.

26. Tilg H, Moschen AR. Adipocytokines: mediators linking adipose tissue, inflammation and immunity. Nat Rev Immunol 2006;6: 772-83.

27. Delmonico MJ, Harris TB, Visser M, Park SW, Conroy MB, Velasquez-Mieyer P, et al. Longitudinal study of muscle strength, quality, and adipose tissue infiltration. Am J Clin Nutr 2009;90: 1579-85.

28. Goodpaster BH, Carlson CL, Visser M, Kelley DE, Scherzinger A, Harris TB, et al. Attenuation of skeletal muscle and strength in the elderly: The Health ABC Study. J Appl Physiol 2001;90: 2157-65. 29. Baracos VE, Reiman T, Mourtzakis M, Gioulbasanis I, Antoun S. Body composition in patients with non-small cell lung cancer: a contemporary view of cancer cachexia with the use of computed tomography image analysis. Am J Clin Nutr 2010;91: 1133S-7S.

\section{Appendices}

\section{Abbreviations}

AUC, area under the curve; BMI, body mass index; CA 19-9, carbohydrate antigen 19-9; CI, confidence interval; CT, computed tomography; CRP; C-reactive protein; DP, distal pancreatectomy; HR, Hazard ratio; HU, Hounsfield Unit; IMAC, intramuscular adipose tissue content; mGPS, modified Glasgow prognostic score; LY, grade of lymphatic vessel invasion; MST, mean survival time; NE, grade of 
perineural invasion; NLR, neutrophil-lymphocyte ratio; OS, overall survival; PD, pancreaticoduodenectomy; PNI, prognostic nutritional index; PLR, platelet-lymphocyte ratio; PMI, psoas muscle mass index; RFS, recurrence-free survival; ROC, receiver operating characteristic; ROI, region of interest; SD, standard deviation; TLC, total lymphocyte count; TP, total pancreatectomy; V, grade of blood vessel invasion 


\section{Figure Legends}

Fig 1. Cross-sectional computed tomographic images of umbilical level.

A. The bilateral psoas muscle areas were measured by manual tracing.

PMI $=$ cross-sectional area of bilateral psoas muscle $/$ height ${ }^{2}\left(\mathrm{~cm}^{2} / \mathrm{m}^{2}\right)$.

B. Subfascial muscular tissue in the multifidus muscle was precisely traced, with 4 small circles placed on subcutaneous fat away from major vessels.

$\mathrm{IMAC}=$ mean $\mathrm{CT}$ value of ROI of multifidus muscle $(\mathrm{HU}) /$ mean CT value of ROI of subcutaneous fat (HU).

PMI, psoas muscle mass index; CT, Computed Tomography; HU, Hounsfield Unit; IMAC, intramuscular adipose tissue content; ROI, Region of interest

Fig 2. A. Box-and-whisker plot of PMI. B. Box-and-whisker plot of IMAC. C. Correlation of PMI with age. $\quad$ D. Correlation of IMAC with age.

PMI, psoas muscle mass index; IMAC, intramuscular adipose tissue content

Fig 3. Overall survival rates after pancreatic resection according to PMI (A) and IMAC (B), and recurrence-free survival rates after pancreatic resection according to PMI (C) and IMAC (D).

PMI, psoas muscle mass index; IMAC, intramuscular adipose tissue content

Fig 4. Overall survival rates after pancreatic resection for patients with normal/high PMI classified according to IMAC (A), and recurrence-free survival rates after pancreatic resection for patients with normal/high PMI classified according to IMAC (B).

PMI, psoas muscle mass index; IMAC, intramuscular adipose tissue content 
TABLE I. Clinicopathological characteristics of patients with low PMI and normal/high PMI.

\begin{tabular}{|c|c|c|c|}
\hline & $\begin{array}{l}\text { Low } \\
\text { PMI } \\
(n=64)\end{array}$ & $\begin{array}{c}\text { Normal/High } \\
\text { PMI } \\
(\mathbf{n}=166)\end{array}$ & P value \\
\hline Age (years); Mean $\pm \mathrm{SD}$ & $69.3 \pm 8.6$ & $64.8 \pm 10.0$ & .002 \\
\hline Sex; Male / Female & $33 / 31$ & $91 / 75$ & .657 \\
\hline BMI; Mean \pm SD & $20.3 \pm 3.2$ & $22.2 \pm 3.2$ & $<.001$ \\
\hline Albumin $(\mathrm{g} / \mathrm{dl}) ;$ Mean $\pm \mathrm{SD}$ & $4.0 \pm 0.1$ & $4.1 \pm 0.0$ & .122 \\
\hline $\mathrm{CRP}(\mathrm{mg} / \mathrm{dl}) ; \mathrm{Mean} \pm \mathrm{SD}$ & $0.5 \pm 0.1$ & $0.3 \pm 0.1$ & .325 \\
\hline Platelet count $\left(\times 10^{4} / \mu \mathrm{l}\right) ;$ Mean $\pm \mathrm{SD}$ & $23.0 \pm 5.8$ & $21.9 \pm 7.7$ & .278 \\
\hline Total lymphocyte count $(/ \mu \mathrm{l}) ;$ Mean $\pm \mathrm{SD}$ & $1467 \pm 64$ & $1493 \pm 40$ & .726 \\
\hline Neutrophil-lymphocyte ratio; Mean \pm SD & $3.0 \pm 0.2$ & $2.7 \pm 0.1$ & .269 \\
\hline Platelet-lymphocyte ratio; Mean \pm SD & $176 \pm 10$ & $162 \pm 6$ & .220 \\
\hline Modified Glasgow prognostic score; 0 / 1 / 2 & $60 / 2 / 2$ & $153 / 11 / 2$ & .369 \\
\hline Prognostic nutritional index; Mean \pm SD & $47.7 \pm 6.6$ & $48.8 \pm 5.0$ & .192 \\
\hline CA19-9 (U/ml); Mean \pm SD & $335.6 \pm 79.0$ & $228.3 \pm 49.0$ & .249 \\
\hline Tumor size $(\mathrm{cm})$; Mean $\pm \mathrm{SD}$ & $3.1 \pm 1.2$ & $2.9 \pm 1.3$ & .407 \\
\hline $\begin{array}{l}\text { Tumor differentiation; } \\
\qquad \text { Well + Moderately / Poorly differentiated adenocarcinoma }\end{array}$ & $55 / 9$ & $142 / 24$ & .939 \\
\hline Stage (UICC); Ia / Ib / IIa / IIb / III / IV & $1 / 0 / 15 / 45 / 1 / 2$ & $4 / 8 / 62 / 84 / 2 / 6$ & .102 \\
\hline Type of operation; PD / DP / TP & $43 / 19 / 2$ & $112 / 47 / 7$ & .918 \\
\hline Resection curability; R0 / R1 & $57 / 7$ & $133 / 33$ & .109 \\
\hline Postoperative complications; $\mathrm{n}$ (\%) (Clavien Dindo grade $\geq 3$ ) & $6(9.4)$ & $9(5.4)$ & .277 \\
\hline Neo-adjuvant chemotherapy; $\mathrm{n}(\%)$ & $7(10.9)$ & $17(10.2)$ & .877 \\
\hline Adjuvant chemotherapy; $\mathrm{n}(\%)$ & $40(62.5)$ & $133(80.1)$ & .006 \\
\hline
\end{tabular}

BMI, body mass index; CA 19-9, carbohydrate antigen 19-9; CRP, C-reactive protein; DP, distal pancreatectomy; IMAC, intramuscular adipose tissue content; PD, pancreaticoduodenectomy; SD, standard deviation; TP, total pancreatectomy; UICC, Union for International Cancer Control 
TABLE II. Clinicopathological characteristics of patients with high IMAC and normal/low IMAC.

\begin{tabular}{|c|c|c|c|}
\hline & $\begin{array}{c}\text { High } \\
\text { IMAC } \\
(n=142)\end{array}$ & $\begin{array}{c}\text { Normal/Low } \\
\text { IMAC } \\
(\mathbf{n}=\mathbf{8 8})\end{array}$ & Pvalue \\
\hline Age (years); Mean $\pm \mathrm{SD}$ & $69.2 \pm 8.5$ & $60.9 \pm 9.7$ & $<.001$ \\
\hline Sex; Male / Female & $69 / 73$ & $55 / 33$ & .040 \\
\hline $\mathrm{BMI} ;$ Mean \pm SD & $22.1 \pm 3.5$ & $20.9 \pm 2.7$ & .007 \\
\hline Albumin (g/dl); Mean \pm SD & $4.1 \pm 0.0$ & $4.1 \pm 0.0$ & .301 \\
\hline $\mathrm{CRP}(\mathrm{mg} / \mathrm{dl}) ;$ Mean $\pm \mathrm{SD}$ & $0.3 \pm 0.1$ & $0.5 \pm 0.1$ & .260 \\
\hline Platelet count $\left(\times 10^{4} / \mu \mathrm{l}\right) ;$ Mean \pm SD & $22.5 \pm 7.5$ & $21.7 \pm 6.8$ & .447 \\
\hline Total lymphocyte count $(/ \mu \mathrm{l}) ;$ Mean \pm SD & $1518 \pm 43$ & $1433 \pm 54$ & .221 \\
\hline Neutrophil-lymphocyte ratio; Mean \pm SD & $2.8 \pm 0.2$ & $2.8 \pm 0.2$ & .917 \\
\hline Platelet-lymphocyte ratio; Mean \pm SD & $170 \pm 7$ & $159 \pm 8$ & .285 \\
\hline Modified Glasgow prognostic score; 0 / 1 / 2 & $132 / 8 / 2$ & $81 / 5 / 2$ & .888 \\
\hline Prognostic nutritional index; Mean \pm SD & $48.4 \pm 0.5$ & $48.5 \pm 0.5$ & .846 \\
\hline CA19-9 (U/ml); Mean \pm SD & $266.5 \pm 53.2$ & $244.7 \pm 67.5$ & .801 \\
\hline Tumor size $(\mathrm{cm})$; Mean $\pm \mathrm{SD}$ & $3.1 \pm 1.3$ & $2.7 \pm 1.2$ & .043 \\
\hline $\begin{array}{l}\text { Tumor differentiation; } \\
\text { Well + Moderately / Poorly differentiated adenocarcinoma }\end{array}$ & $123 / 19$ & $74 / 14$ & .595 \\
\hline Stage (UICC); Ia / Ib / IIa / IIb / III / IV & $3 / 4 / 45 / 87 / 1 / 2$ & $2 / 4 / 32 / 42 / 2 / 6$ & .146 \\
\hline Type of operation; PD / DP/TP & $99 / 36 / 7$ & $56 / 30 / 2$ & .256 \\
\hline Resection curability; R0 / R1 & $120 / 22$ & $70 / 18$ & .335 \\
\hline Postoperative complications; $\mathrm{n}(\%)$ (Clavien Dindo grade $\geq 3$ ) & $11(7.8)$ & $4(4.6)$ & .339 \\
\hline Neo-adjuvant chemotherapy; $\mathrm{n}(\%)$ & $12(8.5)$ & $12(13.6)$ & .211 \\
\hline Adjuvant chemotherapy; $\mathrm{n}(\%)$ & $96(67.6)$ & $77(87.5)$ & $<.001$ \\
\hline
\end{tabular}

BMI, body mass index; CA 19-9, carbohydrate antigen 19-9; CRP, C-reactive protein; DP, distal pancreatectomy; IMAC, intramuscular adipose tissue content; PD, pancreaticoduodenectomy; SD, standard deviation; TP, total pancreatectomy; UICC, Union for International Cancer Control 
TABLE III. Univariate and multivariate analysis of clinicopathological factors and overall survival following resection of pancreatic cancer. (Cox proportional hazard model.)

\section{Variable}

Univariate

Hazard ratio (95\% CI) P value Hazard ratio (95\% CI) P value

Low PMI

High IMAC

Age $(>70)$

Male gender

$\operatorname{BMI}\left(<20 \mathrm{~kg} / \mathrm{m}^{2}\right)$

$\operatorname{NLR}(>4)$

$\operatorname{PLR}(>300)$

$\operatorname{TLC}(<1000 / \mu \mathrm{l})$

$\mathrm{CRP}(>1.0 \mathrm{mg} / \mathrm{dl})$

mGPS (2 vs 0,1$)$

PNI $(<45)$

CA19-9 (>200 U/ml)

Tumor size $(>2.0 \mathrm{~cm})$

Poorly differentiated tumor

LY 2,3 (vs. 0,1)

V 2,3 (vs. 0,1)

NE 2,3 (vs 0,1)

Nodal metastasis

R1 (vs. R0)

PD/TP (vs. DP)

Postoperative complication

(Clavien Dindo grade $\geq 3$ )

Adjuvant chemotherapy (-) (vs. +)$$
2.347(1.658-3.296)
$$$$
2.337(1.617-3.453)
$$$$
1.410(0.999-1.973)
$$$$
1.362(0.977-1.909)
$$$$
0.960(0.662-1.367) \quad .823
$$$$
1.383(0.877-2.095) \quad .157
$$$$
1.005(0.721-1.400) \quad .977
$$$$
1.104(0.636-1.796) \quad .709
$$$$
1.342(0.722-2.285) \quad .332
$$$$
2.705(0.827-6.478) \quad .092
$$$$
\begin{array}{llll}
1.570(1.038-2.309) & .033 & 1.073(0.675-1.650) & .759
\end{array}
$$$$
\begin{array}{llll}
1.797(1.258-2.535) & .002 & 1.012(0.661-1.530) & .957
\end{array}
$$$$
\begin{array}{llll}
1.696(1.152-2.572) & .007 & 1.010(0.659-1.583) & .963
\end{array}
$$$$
\begin{array}{llll}
1.529(0.924-2.398) & .095 & 1.909(1.090-3.199) & .025^{*}
\end{array}
$$$$
2.311(1.358-3.704) \quad .003 \quad 1.840(1.030-3.113) \quad .040 *
$$$$
1.215(0.834-1.737) \quad .304
$$$$
1.761(1.254-2.492) \quad .001 \quad 1.360(0.919-2.022) \quad .124
$$$$
2.041(1.437-2.943) \quad<.001 \quad 1.567(1.064-2.341) \quad .023 *
$$$$
1.575(1.007-2.373) \quad .047 \quad 2.070(1.259-3.324) \quad .005^{*}
$$$$
\begin{array}{llll}
1.443(0.998-2.136) & .051 & 1.446(0.968-2.203) & .072
\end{array}
$$$$
1.702(0.864-3.016) \quad .117
$$

$1.530(1.033-2.214) \quad .034 \quad 1.793(1.143-2.779)$

$.012^{*}$ 
*Statistically significant.

BMI, body mass index; CA 19-9, carbohydrate antigen 19-9; CI, confidence interval; CRP, C-reactive protein; DP, distal pancreatectomy; IMAC, intramuscular adipose tissue content; LY, grade of lymphatic vessel invasion; mGPS, modified Glasgow prognostic score; NE, grade of perineural invasion; NLR, neutrophil-lymphocyte ratio; PD, pancreaticoduodenectomy; PLR, platelet-lymphocyte ratio; PMI, psoas muscle mass index; PNI, prognostic nutritional index; TLC, total lymphocyte count; TP, total pancreatectomy; $\mathrm{V}$, grade of blood vessel invasion 
TABLE IV. Univariate and multivariate analysis of clinicopathological factors and recurrence-free survival following resection of pancreatic cancer. (Cox proportional hazard model.)

\section{Variable}

Low PMI

High IMAC

Age $(>70)$

Male gender

$\operatorname{BMI}\left(<20 \mathrm{~kg} / \mathrm{m}^{2}\right)$

$\operatorname{NLR}(>4)$

$\operatorname{PLR}(>300)$

$\operatorname{TLC}(<1000 / \mu \mathrm{l})$

CRP $(>1.0 \mathrm{mg} / \mathrm{dl})$

mGPS (2 vs 0,1$)$

PNI $(<45)$

CA19-9 (>200 U/ml)

Tumor size $(>2.0 \mathrm{~cm})$

Poorly differentiated tumor

LY 2,3 (vs. 0,1)

V 2,3 (vs. 0,1)

NE 2,3 (vs 0,1)

Nodal metastasis

R1 (vs. R0)

PD/TP (vs. DP)

Postoperative complication

(Clavien Dindo grade $\geq 3$ )

Adjuvant chemotherapy (-) (vs. +)
Univariate

Multivariate

\section{Hazard ratio (95\% CI) P value Hazard ratio (95\% CI) P value}

$1.136(0.837-1.535) \quad .411$

$1.251(0.927-1.694) \quad .143$

$1.039(0.748-1.426) \quad .816$

$1.413(0.934-2.068) \quad .099$

$1.342(0.863-2.021) \quad .186$

$1.331(0.655-2.396) \quad .402$

$1.015(0.621-1.574) \quad .949$

$1.048(0.566-1.771) \quad .873$

$1.905(0.585-4.521) \quad .250$

$1.493(1.035-2.110) \quad .033 \quad 1.210(0.826-1.735) \quad .321$

$2.043(1.481-2.793) \quad<.001 \quad 1.293(0.899-1.843) \quad .164$

$\begin{array}{llll}1.574(1.109-2.289) & .010 & 1.049(0.719-1.561) & .806\end{array}$

$1.266(0.795-1.923) \quad .307$

$1.969(1.180-3.099) \quad .011 \quad 1.595(0.914-2.634) \quad .097$

$1.288(0.919-1.779) \quad .139$

$\begin{array}{llll}1.641(1.211-2.231) & .001 & 1.057(0.752-1.489) & .748\end{array}$

$2.362(1.713-3.300) \quad<.001 \quad 1.924(1.348-2.782) \quad<.001 *$

$1.737(1.154-2.533) \quad .009 \quad 2.017(1.299-3.043) \quad .002 *$

$1.245(0.906-1.734) \quad .178$

$\begin{array}{llll}1.712(0.923-2.902) & .085 & 1.606(0.851-2.788) & .136\end{array}$

$1.117(0.777-1.571) \quad .542$ 
*Statistically significant.

BMI, body mass index; CA 19-9, carbohydrate antigen 19-9; CI, confidence interval; CRP, C-reactive protein; DP, distal pancreatectomy; IMAC, intramuscular adipose tissue content; LY, grade of lymphatic vessel invasion; mGPS, modified Glasgow prognostic score; NE, grade of perineural invasion; NLR, neutrophil-lymphocyte ratio; PD, pancreaticoduodenectomy; PLR, platelet-lymphocyte ratio; PMI, psoas muscle mass index; PNI, prognostic nutritional index; TLC, total lymphocyte count; TP, total pancreatectomy; $\mathrm{V}$, grade of blood vessel invasion 
Fig 1
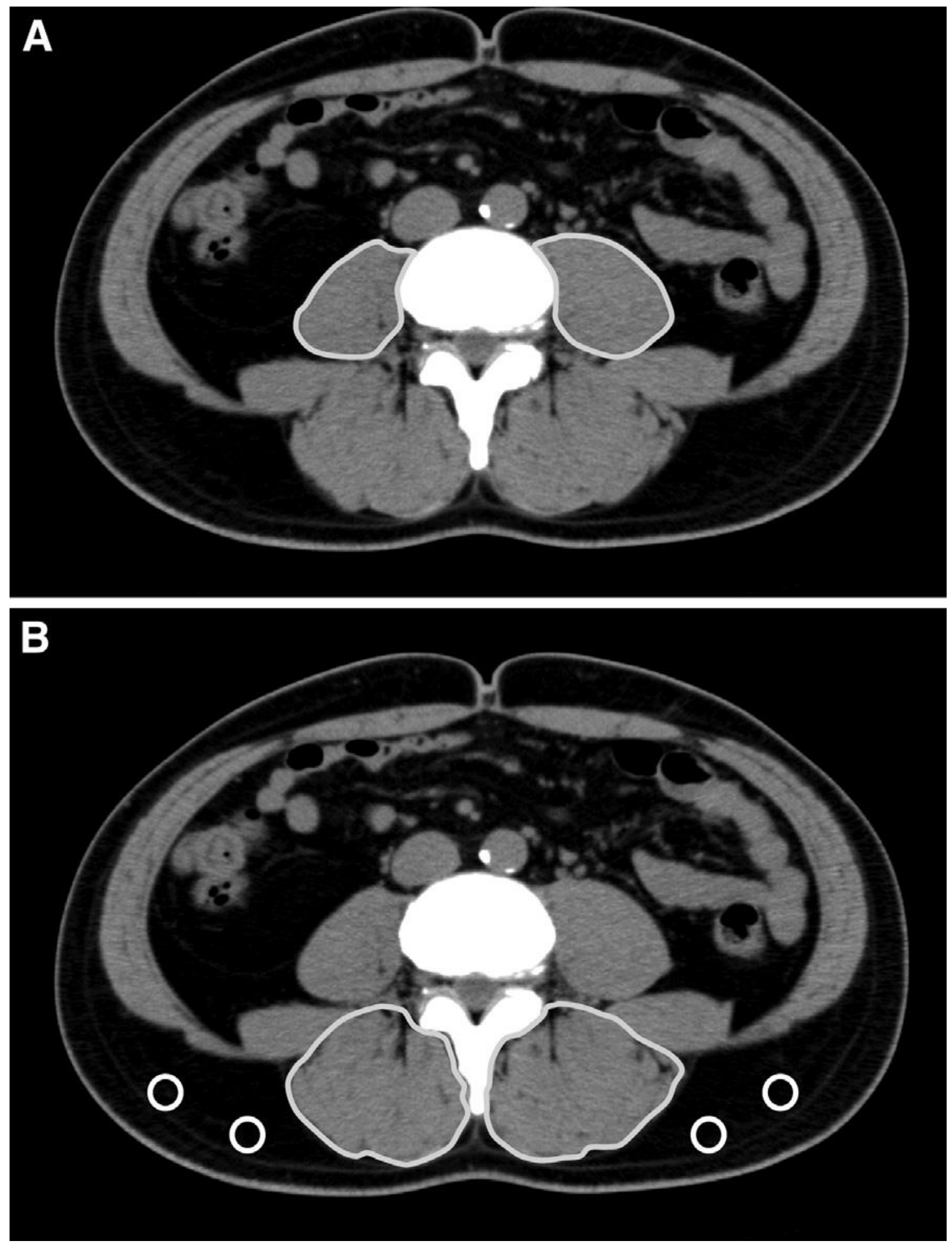
Fig 2
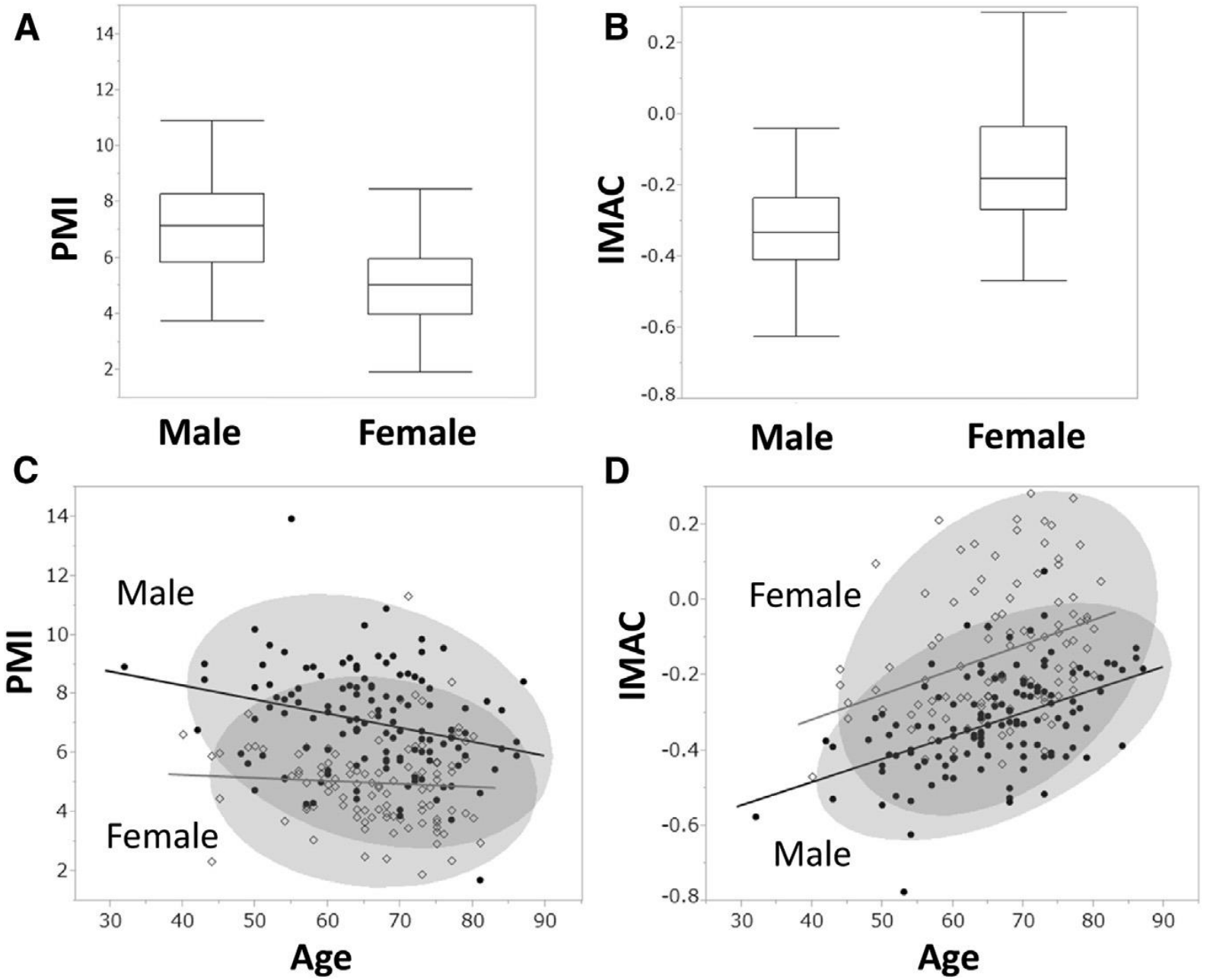
Fig 3
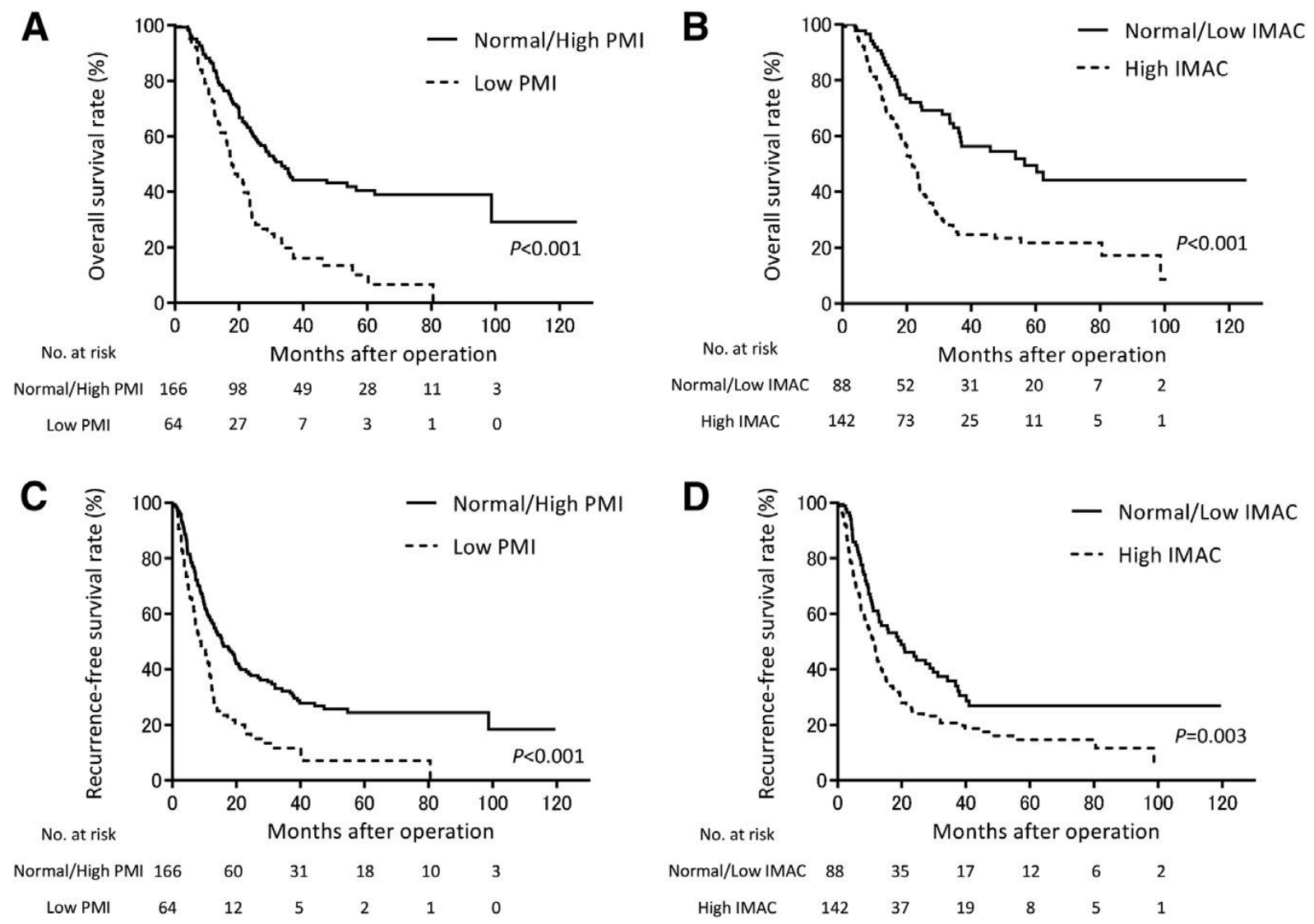


\section{Fig 4}

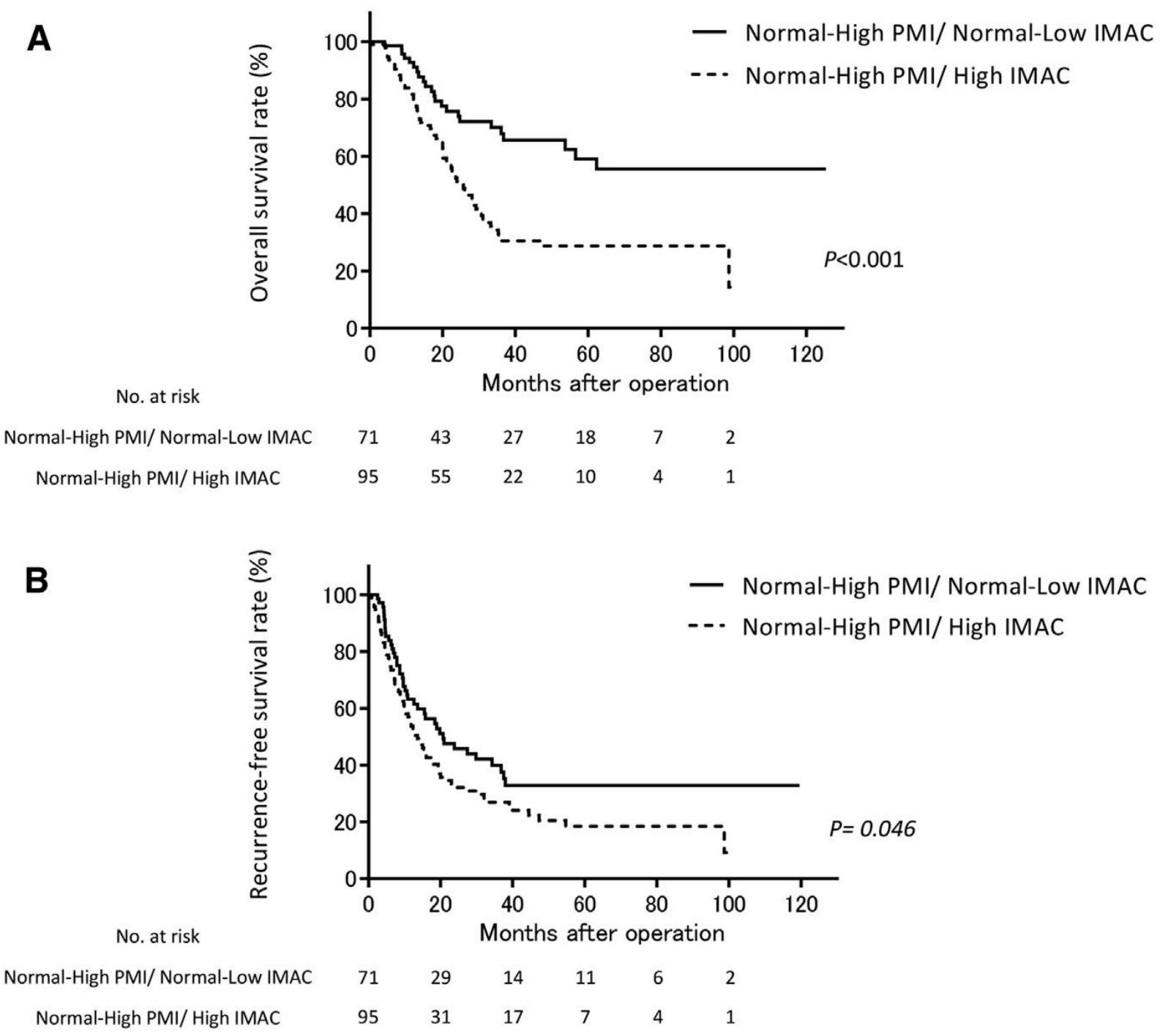

